\title{
Tinkering with extracellular vesicles viruses evolve new infectious units
}

\author{
Mayra Diosa-Toro', Tania Strilets ${ }^{2}$, Shih-Chia Yeh $^{3}$, Mariano A. Garcia-Blanco ${ }^{2,4,5,6}$ \\ ${ }^{1}$ Department of Biomolecular Health Sciences, Faculty of Veterinary Medicine, Utrecht University, The Netherlands; ${ }^{2}$ Department of Biochemistry \\ and Molecular Biology, University of Texas Medical Branch, Galveston, TX, USA; ${ }^{3}$ Cancer Science Institute of Singapore, National University of \\ Singapore, Singapore; ${ }^{4}$ Department of Internal Medicine, University of Texas Medical Branch, Galveston, TX, USA; ${ }^{5}$ Institute of Human Infections \\ and Immunity, University of Texas Medical Branch, Galveston, TX, USA; ${ }^{6}$ Programme in Emerging Infectious Diseases, Duke-NUS Medical \\ School, Singapore, Singapore \\ Correspondence to: Mayra Diosa-Toro. Department of Biomolecular Health Sciences, Faculty of Veterinary Medicine, Utrecht University, The \\ Netherlands. Email: m.a.diosatoro@uu.nl; Mariano A. Garcia-Blanco. Department of Biochemistry and Molecular Biology, University of Texas \\ Medical Branch, Galveston, TX, USA. Email: maragarc@utmb.edu. \\ Comment on: Kerviel A, Zhang M, Altan-Bonnet N. A New Infectious Unit: Extracellular Vesicles Carrying Virus Populations. Annu Rev Cell Dev \\ Biol 2021;37:171-97.
}

Received: 29 November 2021; Accepted: 16 December 2021; Published: 28 February 2022.

doi: 10.21037/exrna-21-26

View this article at: https://dx.doi.org/10.21037/exrna-21-26

A traditional view of virus biology classifies viruses as either non-enveloped (naked) or enveloped. In naked viruses the infectious unit is composed of the nucleic acid genome encapsidated in a viral protein shell, whereas in enveloped viruses, the outermost layer of the virus particle is composed of a lipid membrane derived from the host cell. Whether released by lysis or budding, free virus particles were considered the canonical infectious units. Nevertheless, the tale of virus dissemination is far more complex than previously anticipated as first reveled by a seminal study on Hepatitis A virus (HAV). In it, it was shown that infectious HAV can be released as naked-free viral particles or cloaked in host-derived membranes, whose surface is devoid of viral proteins and resemble extracellular vesicles (EVs) (1).

It is now clear that a large number of non-enveloped and enveloped viruses exploit $\mathrm{EVs}$ as an alternate mode for virus egress and dissemination, which has led some to propose that virus loaded EVs are distinct infectious units. This proposal is comprehensively reviewed by Kerviel, Zhang and AltanBonnet in Annual Review of Cell and Developmental Biology (2). Clear differences exist between free virus particles and infectious EVs regarding the cellular mechanisms that lead to their biogenesis as well the consequences for virus dissemination and antiviral immunity. Therefore, we are sympathetic to the idea of considering EVs as new infectious units and in this commentary we highlight molecular mechanisms that are likely involved in the biogenesis of EVs and their uptake and how these could be subverted by viruses (Figure 1). We are particularly interested in the phenomenon of RNA sorting during positive sense single stranded RNA [(+)ssRNA] virus infection. Therefore, our commentary focuses on these viruses.

(+)ssRNA viruses carry genomes that are functional mRNAs and thus, translation of viral proteins is accomplished following virus entry and genome release to the cell cytoplasm. In other words, genomes are by themselves infectious, and no additional viral proteins are required to launch viral replication. It is likely that this characteristic has conferred them an evolutionary advantage, and, not surprisingly, they constitute the most diverse type of viral pathogens of plants and animals. (+)ssRNA viruses, include both non-enveloped and enveloped viruses: the traditional non-enveloped viruses are well represented members of the Picornaviridae family, whereas members from the Flaviviridae family are examples of enveloped (+) ssRNA viruses.

Even though the replicative strategies of (+)ssRNA viruses are diverse, a hallmark of all of them is the induction of profound membrane rearrangements to generate viral replication organelles (ROs). ROs are formed in different 


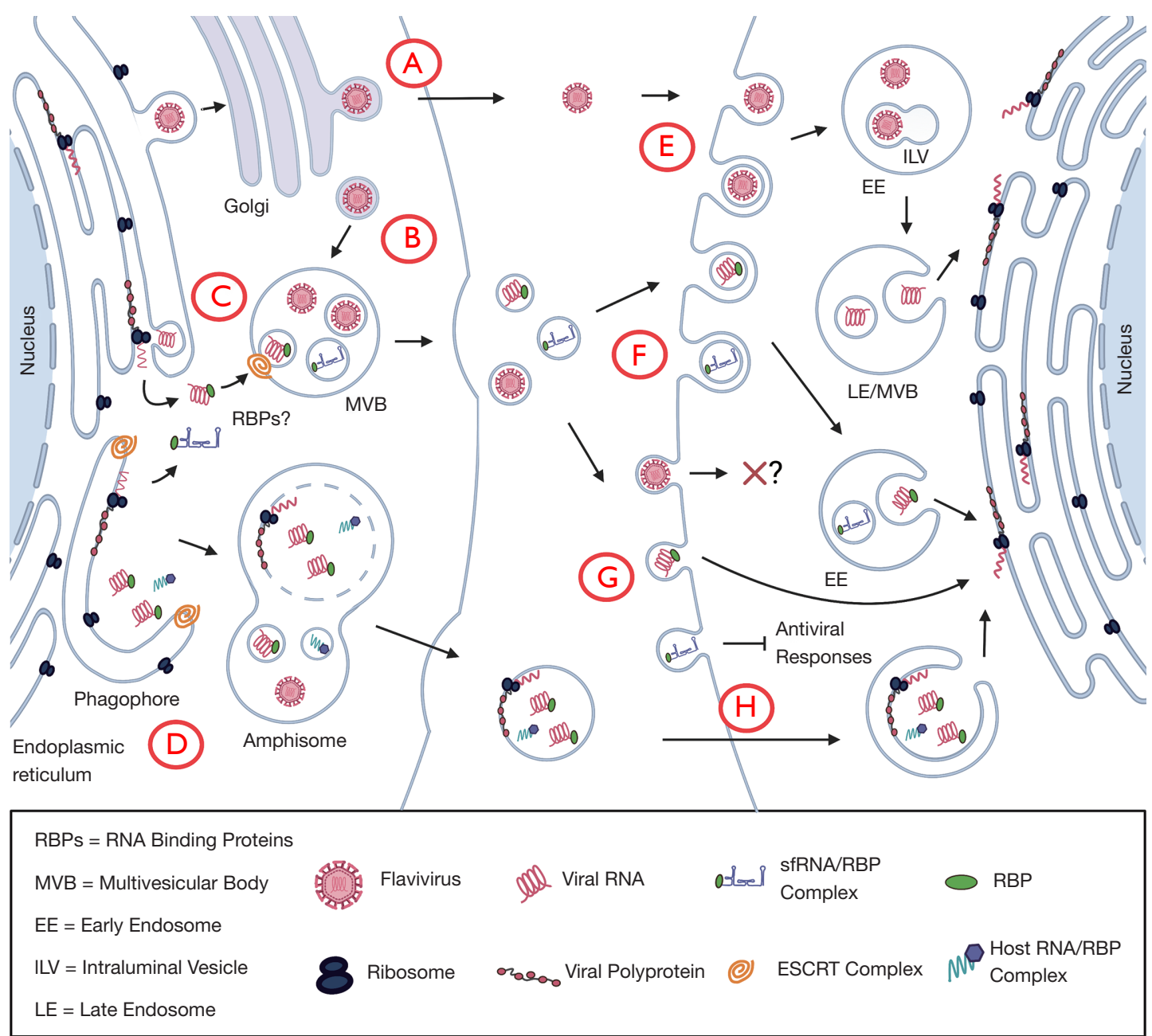

Figure 1 Pathways leading to the release of Flavivirus virions and infectious extracellular vesicles (EVs) and their uptake. (A) Canonical free Flavivirus particles are released via the secretory pathway upon the budding of virions into the lumen of endoplasmic reticulum (ER) and trafficking via the Golgi apparatus to the plasma membrane. Alternatively, viral RNA and ensembled virions are released via the exosomal pathway. We hypothesize that this is the result from (B) fusion of secretory vesicles with multivesicular bodies (MVB) or of (C) recruitment of viral RNA and sfRNA via RNA-binding proteins (RBPs) and endosomal sorting complex required for transport (ESCRT) proteins to late endosomes. Furthermore, (D) Flavivirus infectious EVs are also released via secretory autophagy, whereby, the autophagosome fuses with the MVB (instead of lysosomes) forming amphisomes that then fuse with the plasma membrane. (E) Upon uptake via clathrin mediated endocytosis, canonical Flavivirus particles uncoat and release their viral genomes into the cytosol as endosomes mature. EV packaged virions could also enter via endocytosis, fusing with intraluminal vesicles (ILVs) inside early endosomes to trigger viral uncoating as endosomes mature. This would allow for the release of viral RNA into the cytosol via back fusion of ILVs with the outer endosomal membrane. (F) Endocytosed EV packaged viral/sfRNA could also be released into the cytosol via targeting and fusion at the ER, or lysosomes. (G) EVs containing virions, viral RNA/RBPs, and sfRNA/RBPs could also be delivered by direct fusion with the recipient cell's plasma membrane. This would allow for the expedited delivery of viral RNA to the cytosol to initiate infection and sfRNA to initiate its anti-immune functions. (H) Lastly, vesicles generated from secretory autophagy may be endocytosed or fuse directly with the recipient cell's plasma membrane for delivery of infectious viral RNA and/or proteins. Adapted from "ZIKV Infection Cycle", by Biorender.com (2021). Retrieved from https:// app.biorender.com/biorender-templates. 
subcellular compartments depending on the virus, but the membranous network of the secretory pathway is frequently targeted. The endoplasmic reticulum (ER), the Golgi apparatus and secretory vesicles, including exosomes, lysosomes and secretory amphisomes, are all known sites of (+)ssRNA virus replication (3). ROs concentrate RNA genomes, viral proteins and host factors often involved in lipid biosynthesis and vesicular trafficking; thereby, generating subcellular structures with discernible morphologies of mainly two types: spherule membrane invaginations and double membrane vesicles (DMVs) (4). These ROs are tightly linked to viral assembly sites, where in the case of non-enveloped viruses, the newly synthesized viral RNA genome is encapsidated by the viral capsid protein to assemble a virion. In the case of enveloped viruses, the viral RNA genome is encapsidated to form a viral ribonucleoprotein (RNP) complex, often referred to as nucleocapsid. The nucleocapsid is targeted to cellular membranes enriched with additional viral structural proteins and then buds from the plasma membrane or into intraluminal cellular compartments, thus acquiring a lipid envelop derived from the host membrane in which viral proteins are embedded.

The packaging of viral RNA is complex and has been reviewed recently (5). However, as Kerveil and colleagues recap, viral (+)ssRNA can be found in different forms: (I) in the canonical assembled virions; (II) in EVs that carry fully assembled virions; (III) intriguingly, in EVs carrying infectious genomes (devoid of virions). It is the latter phenomenon we wish to discuss first, by wondering how (+)ssRNA molecules 'escape' targeting to their canonical armor and end up exported from the host cell in EVs.

Although the determinants of viral RNA sorting into EVs remain a black box, the export of cellular RNAs provide a good starting point for hypotheses regarding potential mechanisms involved. Cellular and viral RNA molecules exist in the cell primarily as RNP complexes and thus, RNA-binding proteins (RBPs) are key factors in RNA localization and function. Indeed several RBPs have been implicated in the secretion of cellular RNAs (particularly miRNAs) via the exosomal pathway and secretory autophagy (6-10). Exosome biogenesis requires the host endosomal sorting complex required for transport (ESCRT). ESCRT proteins induce invagination of the late endosomal membrane away from the cytosol, resulting in the uptake of cellular cargo into intraluminal vesicles (ILVs), thereby forming a multivesicular body (MVB). MVBs later fuse with the lysosome for cargo degradation or with the plasma membrane releasing ILVs as exosomes $(11,12)$. ESCRT-I, ESCRT-II and apoptosis linked gene2 -interacting protein $\mathrm{X}$ (ALIX) are important proteins for cargo selection and inward budding of ILVs, whereas ESCRT-III forms the core fission machinery that severs ILVs from the membrane. More recently, it has also been established that ESCRT proteins are also required for the closure of the autophagosome $(13,14)$, a DMV structure that forms around cytoplasmic contents. Autophagosomes fuse with the lysosome for degradation of their intraluminal contents (process known as macroautophagy) or with MVBs to form amphisomes that later fuse with the plasma membrane delineating the process of secretory autophagy (15).

The ESCRT pathway represents the only cellular machinery able to perform inward curvature of limiting membranes, which is required to form ROs, and fission events essential for membrane budding away from the cytosol, which is required for the release of enveloped virus particles into intraluminal compartments or to the extracellular space. Thus, it is not surprising that ESCRT proteins are commonly usurped by viruses to carry out these processes. In fact, the study of viral proteins that recruit ESCRT components, led to the identification of the domains responsible for this recruitment, now known as viral late domains. These are now recognized in the structural proteins of enveloped viruses such as retroviruses, arenaviruses, filoviruses, and rhabdoviruses (16). By engaging ESCRT proteins, late domains promote virus particle release, a mechanism well characterized at the molecular level for enveloped viruses that bud from the plasma membrane (17). Interestingly, release of the enveloped form of HAV (eHAV) also depends on several ESCRT proteins and its adaptors, and two late domains have been identified in the HAV VP2 capsid protein $(1,18)$. ESCRT proteins have also been involved in the assembly of viruses that bud into intracellular compartments, although this involvement is not as well characterized. For example, the ESCRT proteins CHMP2 and 4 function in the membrane deformation that leads to the budding of Dengue virus (DENV) into the lumen of the ER, but ESCRT proteins are dispensable for DENV RNA replication (19). Of note, late domains have not yet been identified in DENV viral proteins and therefore, it is likely that the ESCRT recruitment for DENV budding is mediated by host factors binding either viral proteins or the viral RNA.

Given the function of ESCRT proteins in selecting cargo for EVs (and potentially for autophagosomes), it is temping 
to propose that interaction of ESCRT components with viral RNPs is responsible for their secretion. Indeed, members of ESCRT-II are endowed with RNA-binding activity (20) and an interaction between ESCRT components and viral RNA has been reported during infection with the non-enveloped plant (+)ssRNA tomato bushy stunt virus (21). Alternatively, cellular RBPs could play an important role in sorting viral RNA into EVs by interacting with ESCRT components. For example, the interaction between ALIX and Argonaute 2 has been shown to mediate loading of miRNAs into exosomes (22). Furthermore, EVs released via secretory autophagy, which require at least one ESCRT-III protein (CHIMP4b), are enriched in cellular RBPs, although the full involvement of ESCRT in this pathway remains to be elucidated (10). Interestingly, the non-lytic spread of poliovirus, Coxsakie B3 and enterovirus D68 is attributed to the induction of secretory autophagy [reviewed by (15)] and DENV infectious RNA has been shown to be released in autophagosome-derived vesicles $(23,24)$.

Is it possible that ESCRT proteins recruited to form ROs come in contact with newly synthesized RNA or viral RNPs and this causes their loading into exosomes or amphisomes? Are these 'escape' molecules a product of excess replication and subsequent stochastic cellular processes lead to their secretion? Regardless of the answer, we posit that viruses will evolve to take advantage of these new escape routes and add them to their repertoire. Indeed HAV has evolved two transmission routes, one involving $\mathrm{EV}$-cloaked virions that are found only in blood and thus, mediate intraorganismal transmission; and one via naked virions, which are shed in feces, and thus, mediate interorganismal feco-oral transmission (1). Moreover, arthropod-borne viruses of the Flavivirus genus, such as DENV, Zika virus (ZIKV), and Langat virus (LGTV), provide another interesting case in point of the importance of EVs in enabling viral transmission between organisms.

DENV, ZIKA and LGTV viruses are transmitted between humans through the bite of an infected mosquito or tick, and due to their cycling between human and arthropod hosts, are subject to additional selection pressures. In this context, the advantages of $\mathrm{EV}$-mediated virus transmission, including receptor-independent entry and immune-response evasion, become even more appealing. Interestingly, in human and mosquito cells, as well as in vivo in mosquitoes, EVs carrying infectious Flavivirus genomes are more commonly found than EVs carrying fully assembled virions (25-29). Remarkably, infectious EVs from DENV and ZIKA-infected mosquito cells and LGTV-infected tick cells, were able to transmit infection to mammalian cells $(25,27,28)$. Furthermore, our group has recently uncovered that the subgenomic Flavivirus RNA (sfRNA), which is a key factor modulating DENV infection, is secreted within EVs in the saliva of infected mosquitoes (30). Importantly, the presence of sfRNA in mosquito saliva enhanced virus infectivity in human cells (30). Are infectious EVs a newly identified pathway that facilitates viral dissemination within and among hosts? And if so, how are these infectious EVs delivered to target cells to ensure viral replication?

In this respect, two mechanisms have been proposed for the internalization of cytosolic cargo delivered via EVs: (I) endocytosis of the EV with subsequent delivery of their cargo to endosomes or the ER or (II) fusion at the plasma membrane (31-34). We hypothesize that infectious EVs follow these same two entry mechanisms. Given the preponderance of evidence supporting endocytosis of EVs and the progressively intriguing role of infectious EVs during Flavivirus infection, the remaining of this piece in focus on these viruses (35-37). On their surface, EVs are enriched in both phosphatidylserine (PS) and tetraspanins (38). Their corresponding receptors on recipient cells are TIM/TAMs and integrins, respectively, both of which have been proposed to mediate endocytosis of canonical Flavivirus enveloped particles $(39,40)$. Furthermore, various combinations of $\mathrm{EVs}$ and virions containing tetraspanins and Flavivirus structural proteins have been detected, suggesting a close relationship between Flavivirus virions and EV endocytic entry mechanisms $(28,41)$. Once endocytosed, enveloped Flavivirus particles escape acidifying endosomes to deliver infectious (+)ssRNA into the cytosol, initiating viral translation and RNA synthesis at the ER. If mature virions are packaged into EVs, once endocytosed, viral RNA escape and infection establishment could occur by delivery of virion containing EVs into MVB (31,32). Bis-monoacylglycerol-phosphate and PS are anionic lipids enriched in ILVs and have been demonstrated to assist in fusion of EVs with ILVs and Flavivirus escape from late endosomes/MVBs $(28,42)$. Thus, once endocytosed, virions could escape from EVs by first fusing with ILVs in MVBs, and subsequent back fusion into the cytosol (Figure 1).

On the other hand, infectious viral RNA (likely complexed with host or viral RBPs) and cloaked within EVs could be more efficiently delivered into the cytosol as escape from the endosomal membrane is the only requirement. Once endocytosed, endosomes containing EVs can be targeted to scan the ER, fusing with this membrane or the 
lysosome (31). Given that flaviviruses replicate at the ER, targeting of EV packaged with infectious viral RNA via this route would allow for accelerated initiation of viral replication (Figure 1).

\section{Conclusions}

Viruses have evolved several strategies for their replication and dissemination. In addition to recruiting cellular factors to promote the assembly of free virions, viruses exploit cellular pathways that lead to the biogenesis of EVs. This is not unexpected, when we consider evolution as a tinkering force that will constantly use what already exists (43). Clear advantages emerge from EV-mediated viral dissemination; namely, receptor-independent uptake and en bloc transmission, whereby multiple viral genomes initiate infection in the same cell favoring higher replicative fitness. Furthermore, infectious EV are less likely to be recognized by the host immune system and are more resistant to environmental stresses and disinfection than free particles counterparts (2). With a few exceptions, however, virusinfected cells release both canonical virions and EVenclosed viral clusters/infectious genomes, suggesting that a variety of cellular mechanisms are at play during the late steps of the replication cycle, particularly assembly. What causes the selection of a particular pathway? And how is this relevant for the host? In the well studied example of HAV, release of naked virions is the result of cell death, which translates in tissue pathology, whereas release of EV-cloaked virions, found exclusively in blood, results in evasion of the immune system. What is the proportion of free virions vs infectious EVs in other viral infections and how does it relate to the cell type/tissue being infected? The underlying molecular mechanisms of infectious EV biogenesis and their uptake are far for being understood. Characterizing these mechanisms will certainly influence the way we understand and tackle viral diseases.

\section{Acknowledgments}

Funding: This article is supported by P01 AI150585 (MGB).

\section{Footnote}

Provenance and Peer Review: This article was commissioned by the editorial office, ExRNA. The article has undergone external peer review.
Conflicts of Interest: All authors have completed the ICMJE uniform disclosure form (available at https://exrna. amegroups.com/article/view/10.21037/exrna-21-26/coif). MAGB reports grant funding from NIH/NIAID (USA) P01 AI150585 and funding for his laboratory from UTMB Startup funds. The other authors have no conflicts of interest to declare.

Ethical Statement: The authors are accountable for all aspects of the work in ensuring that questions related to the accuracy or integrity of any part of the work are appropriately investigated and resolved.

Open Access Statement: This is an Open Access article distributed in accordance with the Creative Commons Attribution-NonCommercial-NoDerivs 4.0 International License (CC BY-NC-ND 4.0), which permits the noncommercial replication and distribution of the article with the strict proviso that no changes or edits are made and the original work is properly cited (including links to both the formal publication through the relevant DOI and the license). See: https://creativecommons.org/licenses/by-nc-nd/4.0/.

\section{References}

1. Feng Z, Hensley L, McKnight KL, et al. A pathogenic picornavirus acquires an envelope by hijacking cellular membranes. Nature 2013;496:367-71.

2. Kerviel A, Zhang M, Altan-Bonnet N. A New Infectious Unit: Extracellular Vesicles Carrying Virus Populations. Annu Rev Cell Dev Biol 2021;37:171-97.

3. Paul D, Bartenschlager R. Architecture and biogenesis of plus-strand RNA virus replication factories. World J Virol 2013;2:32-48.

4. Harak C, Lohmann V. Ultrastructure of the replication sites of positive-strand RNA viruses. Virology 2015;479480:418-33.

5. Comas-Garcia M. Packaging of Genomic RNA in Positive-Sense Single-Stranded RNA Viruses: A Complex Story. Viruses 2019;11:253.

6. Villarroya-Beltri C, Gutiérrez-Vázquez C, Sánchez-Cabo F, et al. Sumoylated hnRNPA2B1 controls the sorting of miRNAs into exosomes through binding to specific motifs. Nat Commun 2013;4:2980.

7. Santangelo L, Giurato G, Cicchini C, et al. The RNABinding Protein SYNCRIP Is a Component of the Hepatocyte Exosomal Machinery Controlling MicroRNA 
Sorting. Cell Rep 2016;17:799-808.

8. Shurtleff MJ, Temoche-Diaz MM, Karfilis KV, et al. Y-box protein 1 is required to sort microRNAs into exosomes in cells and in a cell-free reaction. Elife 2016;5:19276.

9. Shurtleff MJ, Yao J, Qin Y, et al. Broad role for YBX1 in defining the small noncoding RNA composition of exosomes. Proc Natl Acad Sci U S A 2017;114:E8987-95.

10. Leidal AM, Huang HH, Marsh T, et al. The LC3conjugation machinery specifies the loading of RNAbinding proteins into extracellular vesicles. Nat Cell Biol 2020;22:187-99.

11. Katzmann DJ, Babst M, Emr SD. Ubiquitin-dependent sorting into the multivesicular body pathway requires the function of a conserved endosomal protein sorting complex, ESCRT-I. Cell 2001;106:145-55.

12. Hanson PI, Cashikar A. Multivesicular body morphogenesis. Annu Rev Cell Dev Biol 2012;28:337-62.

13. Takahashi $Y$, He H, Tang Z, et al. An autophagy assay reveals the ESCRT-III component CHMP2A as a regulator of phagophore closure. Nat Commun 2018;9:2855.

14. Zhou F, Wu Z, Zhao M, et al. Rab5-dependent autophagosome closure by ESCRT. J Cell Biol 2019;218:1908-27.

15. Teo QW, van Leur SW, Sanyal S. Escaping the Lion's Den: redirecting autophagy for unconventional release and spread of viruses. FEBS J 2021;288:3913-27.

16. Votteler J, Sundquist WI. Virus budding and the ESCRT pathway. Cell Host Microbe 2013;14:232-41.

17. Rheinemann L, Sundquist WI. Virus Budding. Encycl Virol 2021:519-28.

18. McKnight KL, Xie L, González-López O, et al. Protein composition of the hepatitis A virus quasi-envelope. Proc Natl Acad Sci U S A 2017;114:6587-92.

19. Tabata K, Arimoto $M$, Arakawa $M$, et al. Unique Requirement for ESCRT Factors in Flavivirus Particle Formation on the Endoplasmic Reticulum. Cell Rep 2016;16:2339-47.

20. Irion U, St Johnston D. bicoid RNA localization requires specific binding of an endosomal sorting complex. Nature 2007;445:554-8.

21. Barajas D, Martín IF, Pogany J, et al. Noncanonical role for the host Vps4 AAA+ ATPase ESCRT protein in the formation of Tomato bushy stunt virus replicase. PLoS Pathog 2014;10:e1004087.

22. Iavello A, Frech VS, Gai C, et al. Role of Alix in miRNA packaging during extracellular vesicle biogenesis. Int J Mol Med 2016;37:958-66.
23. Wu YW, Mettling C, Wu SR, et al. Autophagy-associated dengue vesicles promote viral transmission avoiding antibody neutralization. Sci Rep 2016;6:32243.

24. Li MY, Naik TS, Siu LYL, et al. Lyn kinase regulates egress of flaviviruses in autophagosome-derived organelles. Nat Commun 2020;11:5189.

25. Vora A, Zhou W, Londono-Renteria B, et al. Arthropod EVs mediate dengue virus transmission through interaction with a tetraspanin domain containing glycoprotein Tsp29Fb. Proc Natl Acad Sci U S A 2018;115:E6604-13.

26. Zhou W, Woodson M, Sherman MB, et al. Exosomes mediate Zika virus transmission through SMPD3 neutral Sphingomyelinase in cortical neurons. Emerg Microbes Infect 2019;8:307-26.

27. Zhou W, Woodson M, Neupane B, et al. Exosomes serve as novel modes of tick-borne flavivirus transmission from arthropod to human cells and facilitates dissemination of viral RNA and proteins to the vertebrate neuronal cells. PLoS Pathog 2018;14:e1006764.

28. Martínez-Rojas PP, Quiroz-García E, Monroy-Martínez $\mathrm{V}$, et al. Participation of Extracellular Vesicles from Zika-Virus-Infected Mosquito Cells in the Modification of Naïve Cells' Behavior by Mediating Cell-to-Cell Transmission of Viral Elements. Cells 2020;9:123.

29. Wu SY, Chen YL, Lee YR, et al. The Autophagosomes Containing Dengue Virus Proteins and Full-Length Genomic RNA Are Infectious. Viruses 2021;13:2034.

30. Yeh SC, Tan WL, Chowdhury A, et al. The antiimmune dengue subgenomic flaviviral RNA is found in vesicles in mosquito saliva and associated with increased infectivity. bioRxiv. 2021. doi: https://doi. org/10.1101/2021.03.02.433543

31. Heusermann W, Hean J, Trojer D, et al. Exosomes surf on filopodia to enter cells at endocytic hot spots, traffic within endosomes, and are targeted to the ER. J Cell Biol 2016;213:173-84.

32. Joshi BS, de Beer MA, Giepmans BNG, et al. Endocytosis of Extracellular Vesicles and Release of Their Cargo from Endosomes. ACS Nano 2020;14:4444-55.

33. O'Brien K, Breyne K, Ughetto S, et al. RNA delivery by extracellular vesicles in mammalian cells and its applications. Nat Rev Mol Cell Biol 2020;21:585-606.

34. Parolini I, Federici C, Raggi C, et al. Microenvironmental $\mathrm{pH}$ is a key factor for exosome traffic in tumor cells. J Biol Chem 2009;284:34211-22.

35. Mathieu M, Martin-Jaular L, Lavieu G, et al. Specificities of secretion and uptake of exosomes and other extracellular vesicles for cell-to-cell communication. Nat Cell Biol 


$$
\text { 2019;21:9-17. }
$$

36. McKelvey KJ, Powell KL, Ashton AW, et al. Exosomes: Mechanisms of Uptake. J Circ Biomark 2015;4:7.

37. van Dongen HM, Masoumi N, Witwer KW, et al. Extracellular Vesicles Exploit Viral Entry Routes for Cargo Delivery. Microbiol Mol Biol Rev 2016;80:369-86.

38. van Niel G, D'Angelo G, Raposo G. Shedding light on the cell biology of extracellular vesicles. Nat Rev Mol Cell Biol 2018;19:213-28.

39. Altei WF, Pachane BC, Dos Santos PK, et al. Inhibition of $\alpha v \beta 3$ integrin impairs adhesion and uptake of tumorderived small extracellular vesicles. Cell Commun Signal

doi: 10.21037/exrna-21-26

Cite this article as: Diosa-Toro M, Strilets T, Yeh SC, GarciaBlanco MA. Tinkering with extracellular vesicles viruses evolve new infectious units. ExRNA 2022;4:4.
2020;18:158.

40. Perera-Lecoin M, Meertens L, Carnec X, et al. Flavivirus entry receptors: an update. Viruses 2013;6:69-88.

41. York SB, Sun L, Cone AS, et al. Zika Virus Hijacks Extracellular Vesicle Tetraspanin Pathways for Cell-toCell Transmission. mSphere 2021. [Epub ahead of print] doi: 10.1128/mSphere.00192-21.

42. Zaitseva E, Yang ST, Melikov K, et al. Dengue virus ensures its fusion in late endosomes using compartmentspecific lipids. PLoS Pathog 2010;6:e1001131.

43. Jacob F. Evolution and tinkering. Science 1977;196:1161-6. 